\title{
Natural Orifice Transluminal Endoscopic Surgery for Colorectal Cancer
}

\author{
ISLAM H. METWALLY, M.Sc.*; SHERIF Z. KOTB, M.D.*; MOHAMED A. HEGAZY, M.D.*; \\ WALEED EL-NAHAS, M.D.* and JOSÉ F. NOGUERA, M.D.** \\ Surgical Oncology Unit, Oncology Center, Mansoura University (OCMU), Mansoura, Dakahlia, Egypt* and \\ General \& Digestive Surgery Department, Complejo Hospital Universitario A Coruña (CHUAC), La Coruña, Galicia, Spain**
}

\begin{abstract}
Laparoscopic colorectal resection is used increasingly in colon and rectal cancer treatment. In our study, two NOTES techniques were assessed; laparoscopic colorectal resection with Natural Orifice Specimen Extraction (NOSE) in 16 cases and transanal Total Mesorectal Excision (taTME) in 18 cases. Feasibility, short term oncologic outcome, as well as, comparing both techniques are displayed. Finally, although patients in our study were not randomly assigned to either arm, we could demonstrate better quality of mesorectal excision in the transanal dissection group in comparison to conventional laparoscopy with NOSE technique.
\end{abstract}

Key Words: NOTES - Transanal - Transvaginal - NOSE taTME.

\section{Introduction}

COLORECTAL cancer is the third most common cancer worldwide and the fourth most common cause of cancer death [1]

Surgery was revolutionized at 1987 when Dr. Philippe Mouret performed cholecystectomy through minimal incision using laparoscope [2]

Natural orifice transluminal endoscopic surgery "NOTES", a term coined by the 2005 joint committee of the American Society of Gastrointestinal Endoscopy (ASGE) and the society of American Gastrointestinal and Endoscopic Surgeons (SAGES), has been proposed as an evolutionary step in the ladder of minimally invasive surgery [3]

Interestingly, more than 20 years before the working group white paper on NOTES was published, a pure natural orifice rectal procedure, known as TEM, had already been introduced [4] .

Conventional laparoscopically assisted colorectal resections, unlike many minimally invasive

Correspondence to: Dr. Islam H. Metwally, E-Mail: drislamhany@mans.edu.eg. procedures, still impose an exceptional incisional access burden on the patient in particular; the need for a hand port or a 6-to $10-\mathrm{cm}$ additional incision for specimen retrieval which diminishes much of the advantage offered by the laparoscopic approach. NOTES could solve this problem and provide a significant patients' benefit [4]

The drive to maintain the advantages of laparoscopy and reduce wound-related complications led to Natural OrifiCe Specimen Extraction (NOSE) as an early step in hybrid NOTES techniques [5].

Despite early enthusiasm, NOTES has sometimes been criticized for breaching an otherwise intact and uninvolved organ. A transanal NOTES approach for incisionless colorectal surgery, however, makes sense because a well-planned colotomy used to access the peritoneal cavity can be incorporated into the subsequent anastomosis [6] .

As a "proto-NOTES" platform, the TEM device has many desirable features. It maintains a continuous pressure-regulated pneumo rectum / peritoneum, has four working ports including one for a stabilized laparoscope or flexible endoscope, has specially modified rigid laparoscopic instruments, and is reusable [7].

NOTES can be classified to pure procedures and hybrid procedures. Hybrid procedures such as those assisted by laparoscopy are the result of the

\footnotetext{
Abbreviations:

NOTES : Natural Orifice Transluminal Endoscopic Surgery. NOSE : Natural Orifice Specimen Extraction.

taTME : Transanal Total Mesorectal Excision.

TEM : Transanal Endoscopic Microsurgery.

TEO : Transanal Endoscopic Operation.

ISR : Intersphincteric Resection.

CAP : College of American Pathologist.
} 
laparoscopy and interventional endoscopy evolution to NOTES. Pure procedures impose major obstacles to the safe execution of these procedures in humans. Limitations such as safe full-thickness closure of viscerotomies, absence of triangulation of instruments, and adequate tissue retraction are overcome with the use of the laparoscopic/minilaparoscopic instrument assistance [8].

Currently, there is small number of studies addressing the feasibility and short term outcomes of the different NOTES techniques in colorectal cancer, unfortunately most of these studies are retrospective with no conclusive results.

\section{Patients and Methods}

A prospective interventional study was conducted at the Surgical Unit of the Oncology Center Mansoura University and Complejo Hospital Universitario De A Coruña in Spain, during the period between November 2014 and November 2017 (a total period 35 months), in which 34 patients with localized colorectal cancer were treated using hybrid NOTES technique. They were classified into 2 groups:

Group I: Included 16 patients for whom resection \pm TME was done by conventional laparoscopy with transanal or transvaginal specimen extraction (NOSE).

Group II: Included 18 patients for whom resection with total mesorectal excision was done by laparoscopic assisted transanal resection (taTME).

Patients were followed-up for a median 6.5 months.

Inclusion criteria: Medically fit patient with pathologically proved colorectal cancer.

Exclusion criteria: Patients with American society of Anesthesiologist (ASA) score 4 and 5, patients with cardiac or chest problems that cannot withstand $\mathrm{CO}_{2}$ insufflation, patients with unresectable tumors (defined as those who cannot be resected without a high likelihood of leaving microscopic or gross residual disease at the local site because of tumor adherence or fixation) and patients with obstructed or perforated colorectal cancer.

All patients involved in the study were consented and informed by the potential benefits and possible side effects of the procedure and a written consent was obtained.
Surgical technique:

NOSE group:

Conventional laparoscopic resection was done, and then the specimen was extracted through natural orifice (anal orifice or vaginal orifice).

\section{taTME group:}

Perineal phase: TEO proctoscope with a diameter of $4 \mathrm{~cm}$ was inserted into the rectum, and $\mathrm{CO} 2$ pneumorectum was established. The lumen was occluded using a circumferential purse-string suture below the level of the tumor. About $1 \mathrm{~cm}$ distal to the occluding purse-string, a circumferential incision was done using cautery and angled TEO forceps. The avascular "oncologic" presacral plane was entered posteriorly, and dissection proceeds cephalad using cautery and $\mathrm{CO} 2$ insufflation, in the total mesorectal excision planes. Next, lateral and anterior retrograde dissection was performed, and the abdominal cavity was carefully entered at the peritoneal reflection. Then mobilization is continued up to the sacral promontory. The rectal stump then was reflected into the abdominal cavity, and retrograde dissection was performed until the procedure was limited by instrument length.

Laparoscopic phase: Colon mobilization, lymph node dissection, and mesenteric excision were performed laparoscopically. Mobilization of the splenic flexure was done if needed.

Specimen retrieval: The specimen was delivered transanally and proximal margin divided extracorporeally if mesentery length permits or intracorporeally. For anastomosis, an EEA (end to end anastomosis) stapler anvil was introduced into the proximal colon using a standard purse-string suture with the suture tail left long for later manipulation and connection to the EEA stapler handle. After re-establishment of the pneumorectum, a pursestring suture was placed at the end of the open rectum. Appropriate orientation of the colon was visualized, and the previously placed proximal anvil was delivered into the rectal stump using the suture tail as a handle. The stapler was mated with the anvil shaft under direct vision of a laparoscope. The stapler was brought into opposition, fired, and removed. Pneumorectum was re-established, and the anastomosis was endoluminally inspected.

\section{Statistical analysis:}

The data of the patients were analyzed and statistical values were obtained using Statistical Package for Social Sciences (SPSS) Version 22 (Inc., Chicago, IL). Continuous variables are presented as mean and standard deviation when sym- 
metrical or median and range when asymmetrical. Categorical variables are presented as proportions. Bivariate analysis was done using Student's $t$-test, Mann-Whitney test and Chi-Square test.

\section{Results}

\section{Demographic data:}

From the 34 patients selected for this study, there were 17 males and 17 females; with a mean age of $57 \pm 13$ years (range from 33 to 80 years).

The most common presentation was bleeding per rectum which occurred in 26/34 (76.47\%) of the patients. Pre-operative median serum Carcinoembryonic Antigen (CEA) was 3.3IU with minimum value 0.1 and maximum value 68.1 . Four patients had associated co-morbidities; Hepatitis $\mathrm{C}$ was encountered in $2 / 34$ patients $(5.88 \%)$, one of them with previous radiofrequency ablation for hepatic carcinoma on top, Diabetes Mellitus (DM) in $2 / 34$ patients $(5.88 \%$ ), one of the patients was both diabetic and hepatic, while a history of cerebral stroke with resultant quadriparesis was present in 1 patient. The overall most common tumour site was rectum occurring in $28 / 34(82.35 \%)$ of the patients, the sigmoid was affected in 5/34 (14.7\%) of the patients and the ascending colon was affected in 1 patient $(2.9 \%)$. The median distance of the tumor from the anal verge was $6 \mathrm{~cm}$. The median tumor size at presentation was $3.3 \mathrm{~cm}$. As visualized during colonoscopic examination the polypoid tumor form was the commonest, followed by deeply infiltrating and ulcerative tumours.

\section{Operative data:}

One patient with ascending colon cancer $(2.9 \%)$ underwent right hemicolectomy, 5 patients with sigmoid colon cancer (14.7\%) underwent sigmoidectomy, 20 patients with rectal cancer (58.8\%) underwent low/ultralow anterior resection with total mesorectal excision, and 8 patients with low rectal cancer $(23.5 \%)$ underwent partial ISR.

In the taTME group, transanal approach was used as a first step before the abdominal approach in 3 cases (28.6\%), laparoscopic approach was used as a first step in one case and the combined approach was used in 14 cases.

In the NOSE group, transanal route was used successfully for specimen extraction in 9/16 $(56.25 \%)$ cases and failed in another 4 cases, while in $3 / 16(18.75 \%)$ cases the transvaginal route was used for extraction. In the taTME group the specimen was extracted transanally in 5/18 (27.78\%) cases, and through Pfannenstiel incision in 13/18 $(72.2 \%)$ cases because of bulky rectum.

No stoma was constructed in 7 (20.59\%) patients, while in 20 patients (58.8\%) an ileostomy was done, in $2(5.9 \%)$ patients a transverse colostomy and in $5(14.7 \%)$ patients a Hartmann's colostomy. The anastomosis was hand-sewn in 11 patients; double stapling in 3 patients, triple stapling in 1 patient, single stapling in 14 patients, while no anastomosis was done in 5 patients. In most cases (28) the anastomosis was end to end, while in 1 patient with ascending colon cancer a side to side anastomosis was done. Open conversion was needed in only 4 cases $(11.76 \%)$.

\section{Pathologic data:}

In the 21 patients who received neoadjuvant therapy the response was classified according to CAP system as; no or minimal in 7 cases $(33.3 \%)$, moderate in other $7(33.3 \%)$, marked in $4(19 \%)$, and complete response with no residual tumour in 3 patients (14.3\%). The post-operative TNM staging for them were as follows; stage 0 ( 3 cases $=14.3 \%$ ), stage I ( 2 cases $=9.5 \%)$, stage II ( 7 cases $=33.3 \%)$, stage III $(8=38.1 \%)$, and stage IV $(1=4.8 \%)$.

The overall median number of harvested lymph nodes was 12; ranged between 1 and 26. In 16 cases $(47 \%)$ nodal metastasis was detected pathologically. R0 resection was done in 31 cases (91.2\%). Distal margin was free in 33 cases $(97 \%)$ and the circumferential margin was free in 32 cases $(94.1 \%)$.

\section{Feasibility outcome:}

The mean operative time of the NOSE technique was about 35 minutes shorter than that of the taTME technique. The median time to tolerate oral fluid intake was nearly equal in both groups. While, the median hospital stay was one day shorter in the NOSE group (8.5 versus 9.5 days).

There were 21 complications in 17/34 (50\%) of the patients as follows; wound infection in 6 cases $(17.6 \%)$, major bleeding necessitating reexploration in 2 cases, anastomotic dehiscence in 2 cases, pelvic abscess in 2 cases, intestinal obstruction in 2 cases, urine retention in 2 cases (5.9\% each), and pelvic hematoma in 1 case $(2.9 \%)$. Non-surgical complications occurred in the form of pulmonary embolism, hepatic insufficiency, and metabolic disorder in 4 cases $(11.76 \%)$.

Late post-operative complications occurred in 5 cases $(14.7 \%)$ and were more in the NOSE group. All were anal stenosis with two treated by resection re-anastomosis and three treated by anal dilatation. 
Three out of five cases ended with a permanent stool diversion.

The overall incidence of reoperation/ intervention in our study groups was 14 procedures in 10 patients (29.4\%) as follows; debridement and anastomosis revision for 2 cases (5.9\%) of anastomotic dehiscence, resection-anastomosis for 2 cases $(5.9 \%)$ with anal stenosis, anal dilatation under anesthesia for other $3(8.8 \%)$ cases with anal stenosis, drainage for 3 cases $(8.8 \%)$ of pelvic abscess/ hematoma, and emergent re-exploration for 4 cases $(11.8 \%)$ (two due to major bleeding and two due to intestinal obstruction). Wound infections were treated successfully by conservative care in six cases without intervention.

The NOSE procedure failed in 4 patients. In the four cases 3 were supplemented by Pfannenstiel incision while one was shifted to a conventional open procedure through a midline incision. On the other hand, the taTME procedure failed in 5 patients, in 3 cases it was shifted to conventional up to down laparoscopy (i.e; dissection of the mid/low rectum was completely accomplished by laparoscopy rather than transanally), while in 2 patients it was shifted to open surgery.

\section{Oncological outcome:}

Local recurrence occurred in 1 case $(2.9 \%)$, while distant relapse was not encountered during the study. Also, one case died during follow-up in the taTME group after one and half year without evidence of relapse.

As regard Quirke completeness of mesorectal excision, there were 8 complete, 6 near-complete, and 4 incomplete cases in the taTME group. While in the NOSE group out of the 10 patients with rectal cancer, the quality of mesorectal excision was near complete in 5 cases and incomplete in the other 5 cases.

\section{Comparing NOSE to taTME:}

When comparing the outcome of both groups after excluding the colon cancer (ascending colon and sigmoid colon) cases from the NOSE group; we found no statistical significance as regard feasibility outcomes (operative time, morbidity and blood loss). However, as regard the oncological outcome the quality of mesorectal excision was significantly better in the taTME group, while the number of retrieved node did not differ between both groups (Table 1).

Table (1): Demographic and operative data in the studied cases.

\begin{tabular}{llll}
\hline & NOSE & taTME & $p$-value \\
\hline Number of cases & 10 & 18 & \\
Operative time (minutes) & $309 \pm 61.7$ & $314.44 \pm 89.9$ & .866 \\
Blood loss (ml) & $300(200-750)$ & $500(200-1500)$ & .275 \\
Morbidity (number of cases) & 6 & 9 & .611 \\
Time to oral (days) & $4.5(1-7)$ & $2(1-6)$ & .013 \\
Hospital stay (days) & $10.5(6-45)$ & $9.5(30)$ & .555 \\
Open conversion (number of cases) & 1 & 3 & .172 \\
Quality of TME: & & & \\
$\quad$ Complete & 0 & 8 & .04 \\
$\quad$ Near complete & 5 & 6 & .146 \\
$\quad$ Incomplete & 5 & 4 & \\
Number of retrieved nodes & $5.5(1-26)$ & $12(6-21)$ & \\
Incontinence: & & & \\
$\quad$ Grade II & 4 & 0 & \\
$\quad$ Grade III & 2 & 0 & \\
Grade IV & 1 & 0 & \\
Grade V & 1 & &
\end{tabular}

\section{Discussion}

NOTES had aroused as a pliable option for colorectal cancer. In this study we studied two different natural orifice surgery techniques; the first is the NOSE technique which represents an option to eliminate the scar in colorectal surgery providing superior cosmesis, in addition to less postoperative pain, early return of gastrointestinal function, and early ambulation [9], while the second 
is the taTME technique representing a solution for difficult laparoscopic dissection in a narrow pelvis [10].

In the present study, neoadjuvant therapy was used in 21 cases $(61.76 \%)$ of colorectal cancer based on the stage and plan for resection. This is in concordance with Chi et al., and Kuo et al., who used neoadjuvant chemo-radiotherapy according to the stage of the rectal cancer, in a range varied between $30-100 \%$ [11,12]. According to CAP tumor regression grade, 7 patients $(33.3 \%)$ of our study groups showed good response while 14 (66.7\%) showed poor response to neoadjuvant chemoradiotherapy. Penna et al., in their study reported $38 \%$ complete/marked response and $29 \%$ moderate response, a figure much better than response in our cases [13]. Also, 3 out of the 7 patients with evident down-staging in our study developed complete pathological response (pCR). The previously reported results for complete pathological response after neoadjuvant CRT ranged between $14.5 \%$ and $22 \%[14,15]$.

In our patients, the histopathological examination of the resected specimen showed that 25/34 $(73.5 \%)$ of the patients had well and moderately differentiated, while $8 / 34$ (23.5\%) had poorly differentiated and mucinous adenocarcinomas. This is consistent with grade distribution in similar studies where Helbach included $33.75 \%$ well differentiated $56.25 \%$ moderately differentiated and $10 \%$ poorly differentiated carcinomas [16].

Also, the overall median number of harvested lymph nodes was 12 , with nodal metastasis in 16 $(47 \%)$ cases. The nodal metastasis rate was similar to other studies on the taTME [16] and NOSE [17] techniques, however, the number of retrieved nodes was lower in our study.

The overall mean operative time was 297.8 minutes, with the mean operative time in the NOSE group about 35 minutes shorter than that of the taTME group (279.1 versus 314.4 minutes). In comparison to other studies, our mean operative time was slightly longer $[13,18,19]$.

Also, the median blood loss in our cases was $475 \mathrm{ml}$, which was greater than encountered in other studies $[\mathbf{1 3 , 1 8 , 1 9 ]}$, however, only 4 patients required intraoperative blood transfusion. This can be explained by the learning curve especially in cases operated in Egypt, where the technique is still in its infancy.
The median time to tolerate oral fluid intake was nearly equal in both groups (2.5 versus 2 days). While, the median hospital stay was one day shorter in the NOSE group (8.5 versus 9.5 days). In comparison to total hospital stay of 4.8 days and time to bowel movement of 1.2 days in other studies [9].

Local recurrence occurred in 1 case in the NOSE group, while in the taTME group in a median 8 months of follow-up we did not encounter any local recurrence with single mortality reported 1.5 years after resection not related to cancer relapse. In comparison to Lacy's series on taTME with a longer mean follow-up period of 15 months, he detected $2.3 \%$ local recurrence rate and a $7.6 \%$ rate of systemic recurrence [20].

$\mathrm{R} 0$ resection (i.e. complete resection with no/ zero microscopic residue) was done in $91.18 \%$ of the cases. Distal margin was infiltrated in only one case in the NOSE group, while the circumferential margin was infiltrated ( $<1 \mathrm{~mm}$ free) in 2 cases, one in each group. Indeed, the distal resection margin can be identified early and more accurately during the transanal dissection. In Buchs et al., experience, even when dealing with low tumours, they could found a very good distal margin $(26.9 \mathrm{~mm})$ [21]

Finally, in comparison of the two NOTES techniques in our study for cases with rectal cancer (28 cases); our study found no statistically significant difference between both groups NOSE and taTME as regard feasibility outcomes (operative time, morbidity and blood loss). However, as regard the oncological outcome the quality of mesorectal excision was significantly better in those who underwent taTME in comparison to rectal cancer patients resected using conventional laparoscopy supplemented with NOSE ( $p$-value $=0.04$ ), while the number of retrieved nodes did not differ between both groups. To our knowledge, this the first time to compare these two NOTES techniques, however, limitations do exist as cases were not randomly assigned to either arm.

\section{Conclusion:}

Natural orifice surgery is a promising technique in colorectal oncology. NOSE technique is suitable for most of the cases of laparoscopic rectosigmoid resections, while taTME is suitable for low $/ \mathrm{mid}$ rectal cancer patients. The quality of TME is significantly higher with the taTME technique in comparison to conventional laparoscopic resection with NOSE. 


\section{References}

1- HAGGAR F.A. and R.P. BOUSHEY: Colorectal cancer epidemiology: Incidence, mortality, survival, and risk factors. Clinics in colon and rectal surgery, 22 (04): p. 191-7, 2009.

2- PASRICHA P.J.: A Gastroenterologist's Perspective. Gastrointestinal Endoscopy Clinics, 17 (3): p. 611-6, 2007.

3- BOWMAN D.E.: ASGE/SAGES working group on natural orifice translumenal endoscopic surgery. Gastrointestinal Endoscopy, 63 (2): p. 199-203, 2006.

4- RIEDER E., et al.: A natural orifice transrectal approach for oncologic resection of the rectosigmoid: An experimental study and comparison with conventional laparoscopy. Surgical Endoscopy, 25 (10): p. 3357, 2011.

5- EMHOFF I.A., G.C. LEE and P. SYLLA: Transanal colorectal resection using natural orifice translumenal endoscopic surgery. Digestive Endoscopy, 26 (S1): p. 2942, 2014.

6- RAYMOND T.M., et al.: Hospital stay and return to full activity following laparoscopic colorectal surgery. JSLS: Journal of the Society of Laparoendoscopic Surgeons, 12 (2): p. 143, 2008.

7- BURGHARDT J. and G. BUESS: Transanal endoscopic microsurgery (TEM): A new technique and development during a time period of 20 years. Surgical Technology International, 14: p. 131-7, 2005.

8- LACY A.M., et al.: Minilaparoscopy-assisted natural orifice total colectomy: Technical report of a minilaparoscopy-assisted transrectal resection. Surgical Endoscopy, 26 (7): p. 2080-5, 2012.

9- HUANG Y.C., et al.: Natural orifice specimen extraction with single-stapling anastomosis for distal colon resection: Feasibility and outcomes. Formosan Journal of Surgery, 50 (1): p. 16, 2017.

10- XU W., et al.: Comparison of short-term clinical outcomes between transanal and laparoscopic total mesorectal excision for the treatment of mid and low rectal cancer: A meta-analysis. European Journal of Surgical Oncology, 42 (12): p. 1841-50, 2016.

11- CHI P., et al.: Laparoscopic transabdominal approach partial intersphincteric resection for low rectal cancer: Surgical feasibility and intermediate-term outcome. Annals of Surgical Oncology, 22 (3): p. 944-51, 2015.

12- KUO L.J., et al.: Intersphincteric resection for very low rectal cancer: Clinical outcomes of open versus laparoscopic approach and multidimensional analysis of the learning curve for laparoscopic surgery. Journal of Surgical Research, 183 (2): p. 524-30, 2013

13- PENNA M., et al.: Transanal total mesorectal excision: International registry results of the first 720 cases. Annals of Surgery, 266 (1): p. 111-7, 2017.

14- KUO L.J., et al.: Is final TNM staging a predictor for survival in locally advanced rectal cancer after preoperative chemoradiation therapy? Annals of Surgical Oncology, 14 (10): p. 2766-72, 2007.

15- SMITH J.D., et al.: Nonoperative management of rectal cancer with complete clinical response after neoadjuvant therapy. Annals of surgery, 256 (6): p. 965-72, 2012.

16-HELBACH M.V., et al.: Transanal total mesorectal excision for rectal carcinoma: Short-term outcomes and experience after 80 cases. Surgical endoscopy, 30 (2): p. 464-70, 2016.

17- HISADA M., et al.: Complete laparoscopic resection of the rectum using natural orifice specimen extraction. World Journal of Gastroenterology: WJG, 20 (44): p. 16707, 2014.

18-ATALLAH S., et al.: Transanal minimally invasive surgery for total mesorectal excision (TAMIS-TME): Results and experience with the first 20 patients undergoing curativeintent rectal cancer surgery at a single institution. Techniques in coloproctology, 18 (5): p. 473-80, 2014.

19- SERRA-ARACIL X., et al.: Hybrid NOTES: TEO for transanal total mesorectal excision: Intracorporeal resection and anastomosis. Surgical Endoscopy, 30 (1): p. 346-54, 2016.

20- LACY A.M., et al.: Transanal total mesorectal excision for rectal cancer: Outcomes after 140 patients. Journal of the American College of Surgeons, 221 (2): p. 415-23, 2015.

21- BUCHS N., et al.: A two-centre experience of transanal total mesorectal excision. Colorectal Disease, 18 (12): p. 1154-61, 2016. 


\section{جراحة سرطان القولون والمستقيم بالمنظار

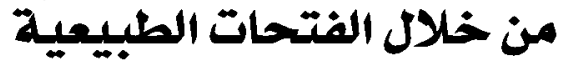

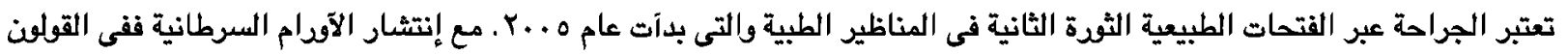

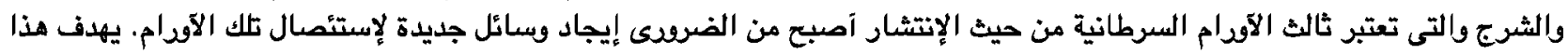

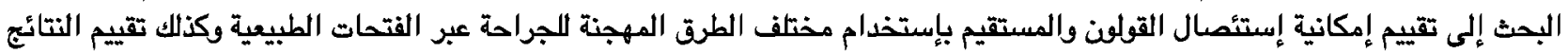

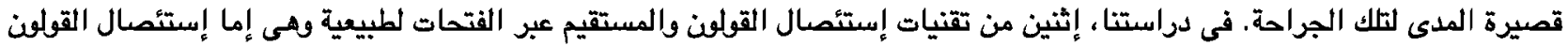

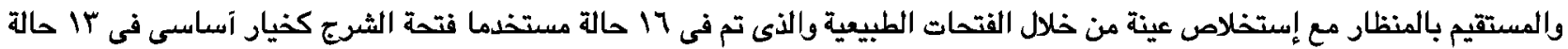

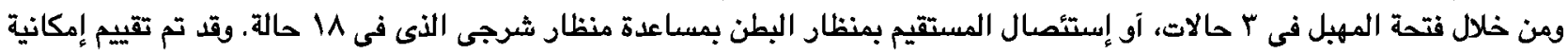

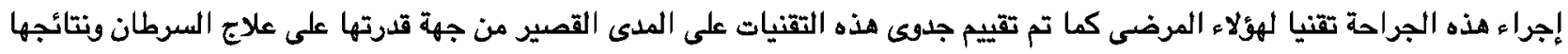
الوظيفية.

وآخيرا، على الرغم من آن المرضى فى دراستنا لم يتم تعيينه عشوائيا إلى آى من الذراعين، فقد تبين لنا آنه قد تم إستئصال المستقيم

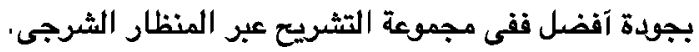

